\title{
Synthesis and Application of Nanocarbon Materials Using Plasma Technology
}

\author{
Fan Yang and Yongfeng Li
}

\begin{abstract}
We report an environmentally friendly approach to the synthesis of ultrasmall monodispersed $\mathrm{Au}, \mathrm{Ag}, \mathrm{Pt}, \mathrm{Pd}$ nanoparticles (NPs) and Pd, Au NPs functionalized oxidation carbon nanotubes (Pd-CNTs, Au-CNTs) by gas-liquid ionic palasma (GLIP) method. Furthermore, the synthesized nanocarbon hybrid materials were characterized by Transmission electron microscopy (TEM) and X-ray diffraction (XRD). The synthesized Pd-CNTs can be applicable for the catalyst in the Suzuki reaction, showing the good reactivity, stability and recyclability, and the Au-CNTs catalyst exhibited much more remarkable catalytic activity in the oxidation of various organosilanes by using water as the solvent compared with other organic solvents (for example THF, ethyl acetate, and acetone), which is very important for organic synthesis from both the standpoint of practical reasons and an economic perspective.
\end{abstract}

Index Terms-Nanocarbon materials, plasma, nanoparticle, molecule transformation reacrions.

\section{INTRODUCTION}

Liquid-related plasmas have recently attracted much attention because of their unique properties such as ultra-high density, high reactivity, high process rate, and preparing nanomaterial at large scale, the plasma technology has been widely utilized for applications in environmental, biomedical, and nanomaterial process fields. On the other hand, this methodology is especially advantageous in avoiding use of toxic stabilizers and reducing agents, and the continuous synthesis, reaction at room temperature and no need to stir during the nanoparticle formation process [1]-[3]. It has been recently a subject of considerable research focused on nanoparticle synthesis by a gas-liquid interfacial plasmas (GLIP) method, including various kinds of nanoparticles such as $\mathrm{Au}, \mathrm{Ag}, \mathrm{Pt}, \mathrm{Pd}$ and $\mathrm{Cu}$ [4]-[7]. However, there still exists a problem that the size distribution and the morphology of the synthesized metal nanoparticles are not controllable, which therefore limits their potential applications. Recently, much effort has been paid to solve these difficulties, such as by using carbon nanotubes, polyvinylpyrrolidone (PVP) or DNA to stabilize the metal nanoparticles. Therefore, it is desirable to develop an efficient and inexpensive synthetic approach for

Manuscript received April 22, 2014; revised June 29, 2014. This work was supported in part by National Natural Science Foundation of China (No. 21202203 and 21106184), the Science Foundation Research Funds Provided to New Recruitments of China University of Petroleum, Beijing (No. YJRC-2013-31 and YJRC-2011-18), and Foundation for the Author of National Excellent Doctoral Dissertation of PR China (No. 201252) and Thousand Talents Program.

Fan Yang and Yongfeng Li are with the State Key Laboratory of Heavy Oil Processing, China University of Petroleum, Changping, Beijing 102249, China (e-mail: yangfan@cup.edu.cn, yfli@cup.edu.cn). the synthesis of metal nanoparticle hybrid materials with controlled morphology, narrow size distribution and wide range of potential applications.

Catalysis using metal nanoparticles has attracted increasing interest due to their potentially green and sustainable catalytic properties. It has been reported that metal NPs supported on various metal oxide surfaces, such as $\mathrm{TiO}_{2}, \mathrm{Al}_{2} \mathrm{O}_{3}, \mathrm{Fe}_{2} \mathrm{O}_{3}$, $\mathrm{SiO}_{2}$ with controllable shape, exhibit unprecedented catalytic activities for transformation of organic substrates. CNTs have many properties for example large chemically active surface, unique physical properties, inherent size, hollow geometry and stability at high temperatures [8], and graphene is a novel two-dimensional material with atoms arranged in a honeycomb lattice, which has exhibited fascinating exceptional electronic, mechanical, and chemical properties due to its unique physical structure and dimensions [9]. Considering these characters, such nanocarbon materials have been normally used as an ideal support for the dispersion and stabilization of metal nanoparticle (NPs).

Based on this background, here we report the synthesis of ultrasmall monodispersed metal NPs (Au NPs, Ag NPs and Pd NPs) and metal NPs functionalized nanocarbon materials by an environmentally-friendly GLIP method. Furthermore, the new catalyst based on nanocarbon materials has been proved to be stable and high catalytic performance in organic molecule transformation reactions [8]-[10].

\section{EXPERIMENTAL}

\section{A. Typical Procedure for the Formation of Metal NPs}

The experimental configuration of ionic liquid introduced plasma, which is used for the synthesis of the metal NPs, is similar to the previous work [8]-[10]. The glow discharge plasma was generated between the top flat stainless steel (SUS) and bottom ionic liquid electrode by using a DC power source (KIKUSUI PMC 500-0.1A). Ar gas was introduced and used as the plasma forming gas, the chamber was a stainless steel with inner diameter of $70 \mathrm{~mm}$ and four glass windows, and the gap between electrodes is $4 \mathrm{~mm}$. A direct current (DC) power source with voltage $V_{\mathrm{DC}}=220-250 \mathrm{~V}$ is applied to a stainless steel electrode in gas phase for the generation of an Ar plasma, where the discharge current $I=$ $0.02 A$ is fixed, and the Ar gas is introduced up to a pressure $P_{\text {gas }}=290 \mathrm{~Pa}$. Oleylamine was dissolved in butyl-3-methylimidazolium tetrafluoroborate by sonication for $15 \mathrm{~min}$, metal salt was added to the mixture sonication for $5 \mathrm{~min}$. The solution was transferred to the stainless steel cell of the experimental setup. The inter-electrode gap is $4 \mathrm{~mm}$. The gas pressure of $\mathrm{Ar}$ in the chamber was evacuated to $290 \mathrm{~Pa}$ by a 
vacuum pump. The discharge current was $0.02 \mathrm{~A}$, and the voltage was in the range of 220-250 V. The plasma was kept steady at this situation for $20 \mathrm{~min}$. The synthesized metal NPs in the ionic liquid were precipitated out by adding the ethanol and centrifugation at $4000 \mathrm{rpm}$ for $10 \mathrm{~min}$, and then the product was washed for four times and dispersed in methylene chloride.

\section{B. Synthesis of the Pd-CNTs Hybrid Materials}

$50 \mathrm{mg}$ oxidation CNTs were dispersed in ethanol by sonicated for $15 \mathrm{~min}$. After that, $\mathrm{Pd}(\mathrm{OAc})_{2}$ ethanol solution were added to the dispersed CNTs in ethanol solution, then the ethanol was evaporated at $40-50{ }^{\circ} \mathrm{C}$. As a result, the $\mathrm{Pd}$ compounds were well dispersed on the surface of oxidation CNTs. The obtained oxidation CNTs decorated with the Pd compounds were dispersed in the ionic liquid 1-butyl-3methylimidazolium tetrafluoroborate $\left([\mathrm{bmim}] \mathrm{BF}_{4}\right)$. For the formation of Pd NPs, electrons were irradiated toward the ionic liquid for $\mathrm{t}=10 \mathrm{~min}$, then the mixture were sonicated in ethanol to remove the excess impurities and extracted from the ionic liquid by a centrifuge process.

\section{Catalytic Activity of the Pd-CNTs}

The Pd-CNTs catalyst, corresponding to a percentage of $\mathrm{Pd}$ of $1 \mathrm{mmol} \%$ with respect to 4-Bromoacetophenone was used, and 4-Bromoacetophenone (0.5 mmol, $100 \mathrm{mg})$, phenylboronic acid $(0.6 \mathrm{mmol}, 73 \mathrm{mg})$ were mixed together in a pressure vial, then $2 \mathrm{~mL}$ potassium carbonate solution $(2 \mathrm{M})$ and $2 \mathrm{~mL}$ ethanol were added, the mixture was stirred at $80{ }^{\circ} \mathrm{C}$ for $3 \mathrm{~h}$. After cooling to room temperature, the mixture was filtered through a polyvinylidene fluoride (PVDF) membrane with $0.2 \mu \mathrm{m}$ pore size in order to isolate the catalysts which were washed with $\mathrm{CH}_{2} \mathrm{Cl}_{2}(10 \mathrm{~mL})$ and water $(10 \mathrm{~mL})$, and dried at $110{ }^{\circ} \mathrm{C}$. The alcoholic/aqueous solution was extracted with diethylether $(10 \mathrm{~mL})$ for 3 times. The organic extracts were dried with anhydrous magnesium sulfate, filtered, and evaporated to dryness, allowing the isolation of pure 1-([1,1-biphenyl]-4-yl) ethanone as the reaction product.

\section{Synthesis of the Au-CNTs Hybrid Materials}

The decoration of AuNPs on the oxidation CNTs catalyst was carried out by a GLIP method with $\mathrm{HAuCl}_{4} \cdot 4 \mathrm{H}_{2} \mathrm{O}$, oxidation CNTs and oleylamine (OA) at room temperature. $50 \mathrm{mg}$ oxidation CNTs were added to the reactor, $\mathrm{HAuCl}_{4} \cdot 4 \mathrm{H}_{2} \mathrm{O}$ were dispersed in 1-butyl-3-methylimidazolium tetra-fluoroborate $\left([\mathrm{BMIM}] \mathrm{BF}_{4}\right)$ and $\mathrm{OA}$, then the Au solution was added to the reactor, and the Au solution and CNTs mixture was left for $10 \mathrm{~min}$. For the formation of AuNPs, electrons were irradiated toward the ionic liquid, and the electron irradiation for $t=15 \mathrm{~min}$, the mixture was sonicated in ethanol to remove the excess impurities and extracted from the ionic liquid using a centrifuge.

\section{E. Catalytic Activity of the Au-CNTs}

The organosilanes in water solution $(0.25 \mathrm{M}, 2 \mathrm{~mL})$ and Au-CNTs $\left(0.1 \mathrm{~mol} \%, 1.3 \mathrm{mg}\right.$ ) were added to $\mathrm{PhMe}_{2} \mathrm{SiH}$ $(78 \mathrm{~mL}, 0.5 \mathrm{mmol})$ at room temperature. The reaction mixture was stirred at room temperature for $40 \mathrm{~min}$ and monitored by thin layer chromatography (TLC) analysis. The mixture was filtered through a polyvinylidene fluoride (PVDF) membrane with $0.2 \mu \mathrm{m}$ pore size in order to isolate the catalysts which were washed with water $(10 \mathrm{~mL})$ and acetone $(10 \mathrm{~mL})$, and dried at $110 \mathrm{C}$. The filtrate was extracted with diethylether three times. The organic extracts were dried with anhydrous magnesium sulfate, filtered, and evaporated to dryness. After concentration, the residue was purified by silica gel chromatography to afford $\mathrm{PhMe}_{2} \mathrm{SiOH}(75.6 \mathrm{mg}, 99 \%)$ as yellowish oil.

\section{RESULTS AND DISCUSSION}

Transmission electron microscopy (TEM) images for Au, Ag, Pt and Pd NPs stabilized by OA prepared with GLIP are shown in Figs. 1(a)-(d), indicating that the monodispersed $\mathrm{Au}$, $\mathrm{Ag}, \mathrm{Pt}$ and Pd NPs were formed, the size distribution of the $\mathrm{Au}, \mathrm{Ag}, \mathrm{Pt}$ and Pd NPs were 2.4, 2.9, 2.0 and $2.7 \mathrm{~nm}$, respectively. High-resolution transmission electron microscopy (HRTEM) images of each kinds of metal NPs are shown in Fig. 1(e)-(h), in which the interfinger distance are measured, and it was corresponding to the (111) of the face centered cubic Au, Ag, Pt and Pd, respectively.
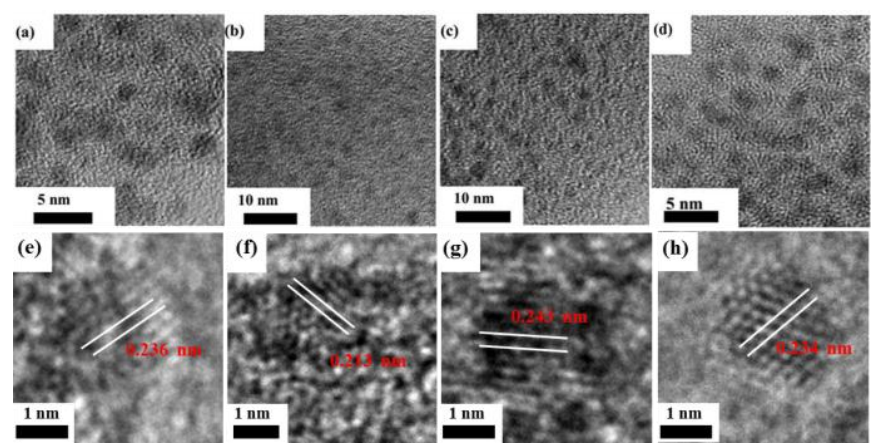

Fig. 1. TEM images of Ag (a), Pt (b), Pd (c), Au (d), and HRTEM images of $\mathrm{Ag}(\mathrm{e}), \mathrm{Pt}(\mathrm{f}), \mathrm{Pd}$ (g), Au (h).
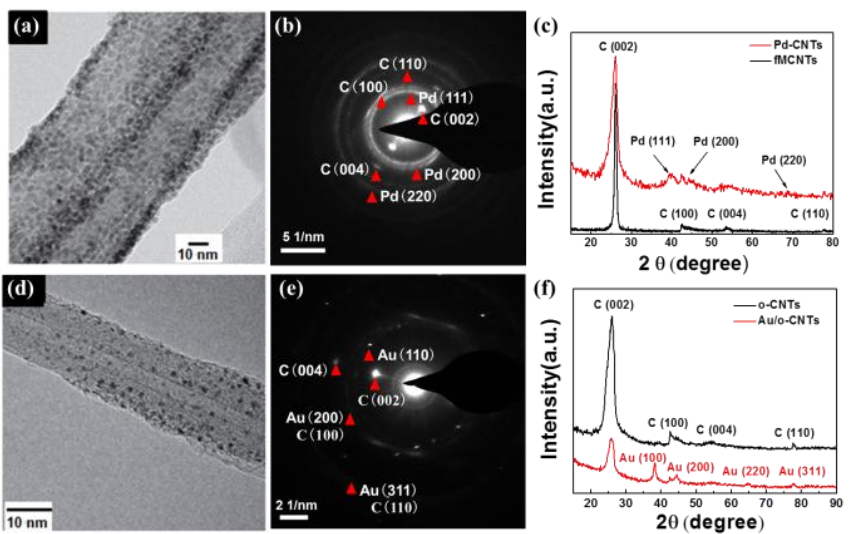

Fig. 2. TEM image of Pd-CNTs (a), SEAD image of Pd-CNTs (b), XRD image of Pd-CNTs (c), TEM image of Au-CNTs (d), SEAD image of Au-CNTs (e), XRD image of Au-CNTs (c).

Furthermore, metal NPs supported on nanocarbon materials have been synthesized by the GLIP method, for example, oxidation CNTs supporting Pd NPs (Pd-CNTs) and $\mathrm{Au}$ NPs (Au-CNTs). Their corresponding TEM images are summarized in Fig. 2(a) and Fig. 2(d), and the results demonstrate that all synthesized nanocarbon materials exhibit uniform morphologies and the particle diameters of Pd-CNTs, Au-CNTs are $3 \mathrm{~nm}, 1.2 \mathrm{~nm}$, respectively. Moreover, the samples are characterized by SEAD, XRD as indicated in Figs. 2(b), 2(c), 2(e), 2(f), showing the characteristic diffraction 
peaks at $25.9^{\circ}, 42.8^{\circ}, 54.3^{\circ}$ and $77.7^{\circ}$, corresponding to (002), (100), (004), and (110) reflections of graphite, respectively. Diffraction peaks were also observed at $40.1^{\circ}, 46.7^{\circ}$, and $68.7^{\circ}$, which could be indexed as the (111), (200), and (220) reflections of crystalline $\mathrm{Pd}(0)$, respectively. The peaks at $38.1^{\circ}, 44.4^{\circ}, 64.5^{\circ}$, and $77.6^{\circ}$ correspond to (111), (200), (220), and (311) reflections of crystalline $\mathrm{Au}$ (0), respectively.

The above results demonstrate that the metal NPs decorated nanocarbons have been successfully prepared, and its application as new catalysts for organic molecule transformation reactions have been further explored. Fig. 3 shows the Pd-CNTs catalyzed Suzuki reactions. The reaction of aryl bromide with phenylboronic acid was carried out in the presence of $\mathrm{Pd}-\mathrm{CNT}$ s of $0.1 \mathrm{~mol} \%$, using $2 \mathrm{M} \mathrm{K}_{2} \mathrm{CO}_{3}$ solution and ethanol as solvent, giving the desired product with $99 \%$ yield at $80{ }^{\circ} \mathrm{C}$. Both aryl bromide bearing an electrondonating and electro-withdrawing aromatic bromide react with phenylboronic acid were subject to the same conditions, the excellent yields were obtained. The coupling of bromobenzene and 1-brormonaphthalene with phenylboronic acid were examined, the corresponding products were obtained in high yields.
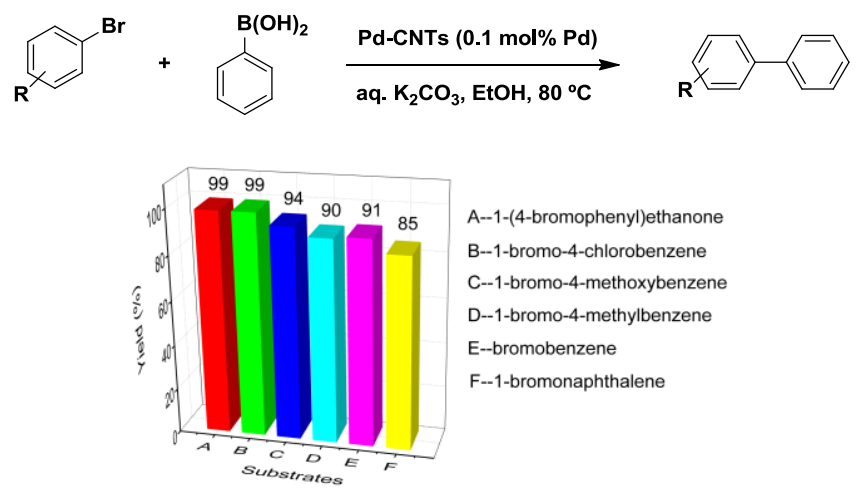

Fig. 3. Yield (\%) of Suzuki-Miyaura reaction with phenylboronic acid catalyzed by Pd-CNTs.

The reusability is the most important feature for a heterogeneous catalyst, which is superior to a homogenous one. First, to confirm the reaction indeed catalyzed by solid Pd-CNTs rather than by homogenous Pd species, we first carried out the reaction, then the catalyst Pd-CNTs was removed by filtration, and the leaving solvent was evaporated and treated with nitrohydrochloric acid. The Pd content was checked by inductively coupled plasma optical emission spectrometry (ICP-OES) analysis, and no leaching of Pd NPs was found during the reaction. To access recyclability of Pd-CNTs, multiple coupling reaction of 4-Bromoacetophenone with phenylboronic acid were carried out by filtration for the separation of the catalyst from the reaction mixture. The catalyst was repeatedly used for four times, the yeilds of the products were shown in Fig. 4.

Furthermore, we performed the oxidation of dimethylphenylsilane with water by using Au-CNTs as a catalyst, the reaction was proceeded smoothly, and the product was obtained in $99 \%$ yield as shown in Fig. 5. In the presence of various organic solvents, including THF, EtOAc and DMF afforded the corresponding silanols in $99 \%$ yield in
$55 \mathrm{~min}, 70 \mathrm{~min}$ and $85 \mathrm{~min}$, respectively. When using toluene as solvent, the reaction cannot proceed. In comparison, the product was surprisingly obtained in $99 \%$ yield as a single product in only $7 \mathrm{~min}$ in $\mathrm{H}_{2} \mathrm{O}$. Usually, heterogeneous catalyzed oxidation of silanes to silanols was more effective by using the organic solvent compared with $\mathrm{H}_{2} \mathrm{O}$ [11].

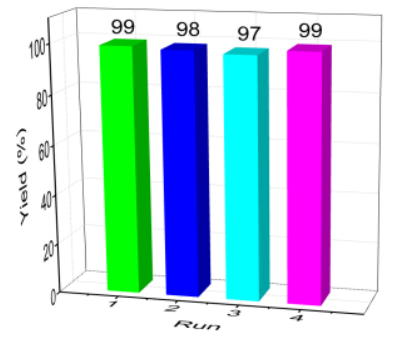

Fig. 4. Yield (\%) of coupling reaction of 4'-bromoacetophenone with phenylboronic acid in subsequnt runs with catalyst.

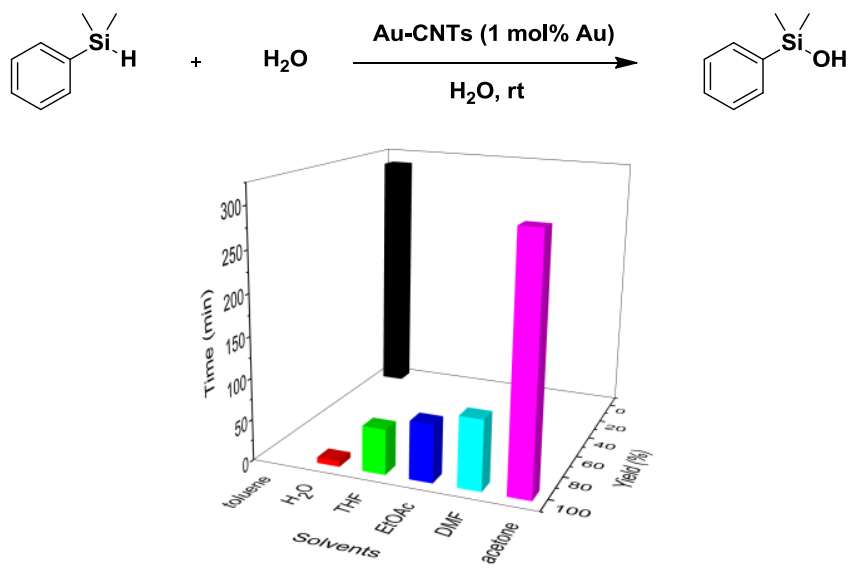

Fig. 5. Oxidation of dimethylphenylsilane with catalyst Au-CNTs in different solvents.

The catalytic oxidation reactions with a variety of organosilanes using the Au-CNTs catalyst were carried out, the results were shown in Fig. 6. First, triethylsilane exposed sterically was quantitatively oxidized to the corresponding silanol in 15 minutes without the formation of disiloxane. Next, the oxidation of two reputedly challenging substrates was examined, the corresponding silanols were obtained in high yields by increasing the catalyst loading amount and the reaction time. The Au-2 catalyst could also be used in the oxidation of diphenylsilane, and the corresponding silanol was obtained in high yield.

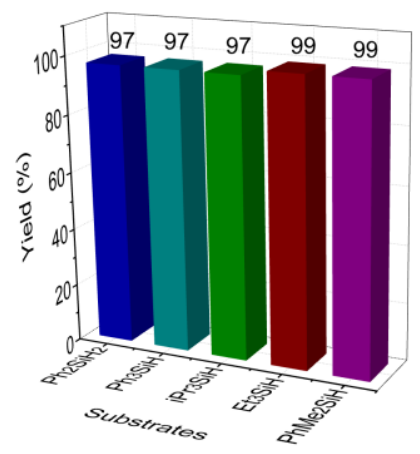

Fig. 6. Yield (\%) of oxidation organosilanes in water catalyzed by Au-2.

The leaching of the AuNPs in the reaction was also examined using an ICP-OES, and no leaching of AuNPs was 
detected by the ICP analysis. To assess recyclability of Au-CNTs, multiple dimethylphenylsilane oxidation cycles were carried out, and the recovery of the heterogeneous catalyst is carried out by filtration for the separation of the catalyst from the reaction mixture. The catalyst was repeatedly used four times, but no significant loss of activity was observed. The product was obtained nearly quantitatively every time as shown in Fig. 7.

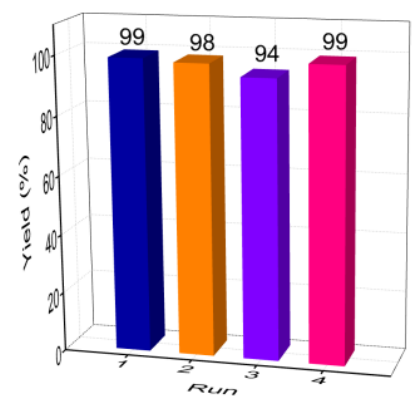

Fig. 7. Yield (\%) of oxidation organosilanes in water in subsequnt runs with catalyst Au-CNTs.

\section{CONCLUSIONS}

In summary, we report the synthesis of ultrasmall monodispersed metal NPs (Au NPs, Ag NPs, Pd NPs) and metal NPs functionalized nanocarbon materials (Pd-CNTs, Au-CNTs), by an environmentally-friendly GLIP method. Furthermore, the new catalyst based on nanocarbon materials has been proved to be stable, and shows high catalytic performance in organic molecule transformation reactions. The synthesized Pd-CNTs can be applicable for the catalyst in the Suzuki reaction, showing the good reactivity, stability and recyclability, and the Au-CNTs are highly efficient heterogeneous catalysts for the selective oxidation of silanes in $\mathrm{H}_{2} \mathrm{O}$.

\section{REFERENCES}

[1] C. Richmonds, M. Witzke, B. Bartling, S. W. Lee, J. Wainright, C.-C Liu, and R. M. Sankaran, "Electron-transfer reactions at the plasma-liquid interface," Journal of the American Chemical Society, vol. 133, pp. 17582-17585, 2011.

[2] M. G. Kong, G. Kroesen, G. Morfill, T. Nosenko, T. Shimizu, J. Van Dijk, and J. Zimmermann, "Plasma medicine: an introductory review," New Journal of Physics, vol. 11, pp. 115012, 2009.

[3] K. Harada and S. Suzuki, "Formation of amino acids from elemental carbon by contact glow discharge electrolysis," Nature, vol. 266, pp. 275-276, 1977.

[4] O. Höfft and F. Endres, "Plasma electrochemistry in ionic liquids: an alternative route to generate nanoparticles," Physical Chemistry Chemical Physics, vol. 13, pp. 13472-13478, 2011.
[5] Q. Chen, T. Kaneko, and R. Hatakeyama, "Rapid synthesis of water-soluble gold nanoparticles with control of size and assembly using gas-liquid interfacial discharge plasma," Chemical Physics Letters, vol. 521, pp. 113-117, 2012.

[6] S. A. Meiss, M. Rohnke, L. Kienle, S. Z. El Abedin, F. Endres, and J. Janek, "Employing plasmas as gaseous electrodes at the free surface of ionic liquids: deposition of nanocrystalline silver particles," Chem Phys Chem, vol. 8, pp. 50-53, 2006.

[7] M. Brettholle, O. Höfft, L. Klarhöfer, S. Mathes, W. Maus-Friedrichs, S. Z. El Abedin, S. Krischok, J. Janek, and F. Endres, "Plasma electrochemistry in ionic liquids: deposition of copper nanoparticles," Physical Chemistry Chemical Physics, vol. 12, pp. 1750-1755, 2009.

[8] F. Yang, Y. F. Li, T. Liu, K. Xu, L. Q. Zhang, C. M. Xu, and J. S. Gao "Plasma synthesis of Pd nanoparticles decorated-carbon nanotubes and its application in Suzuki reaction," Chemical Engineering Journal, vol. 226, pp. 52-58, 2013.

[9] C. Lee, X. Wei, J. W. Kysar, and J. Hone, "Measurement of the Elastic Properties and Intrinsic Strength of Monolayer Graphene," Science, vol. 321, pp. 385-388, 2008.

[10] T. Liu, F. Yang, Y. F. Li, L. Ren, L. Q. Zhang, K. Xu, X. Wang, C. M. $\mathrm{Xu}$, and J. S. Gao. "Plasma synthesis of carbon nanotube-gold nanohybrids: efficient catalysts for green oxidation of silanes in water," Journal of Materials Chemistry A, vol. 2, pp. 245-250, 2014.

[11] W. J. Li, A. Q. Wang, X. F. Yang, Y. Q. Huang, and T. Zhang, " $\mathrm{Au} / \mathrm{SiO}_{2}$ as a highly active catalyst for the selective oxidation of silanesto silanols," Chemical Communications, vol. 48, pp. 9183-9185, 2012.

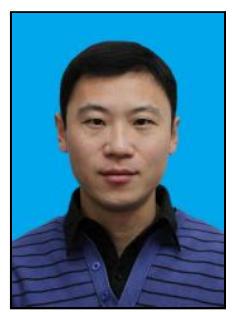

Fan Yang was born on 30 August 1983 at Jilin Province of China, who received his bachelor's degree in the Department of Environment Engineering, Faculty of Environment and Chemical Engineering, Nanchang Hangkong University in Jiangxi, China in 2005. In 2008, he obtained postgraduate degree from Dalian University of Technology in Dalian, China in the field of catalytic chemistry. In 2008 he followed the state scholarship programme, China Scholarship Council, China. In 2012, he received the title of doctor from Chemistry Programme, Tohoku University in Sendai, Japan. He has been a lecturer of Chemical Engineering, State Key Laboratory of Heavy Oil Processing, China University of Petroleum at Beijing form 2012-2013. He has been an associate professor from since 2013

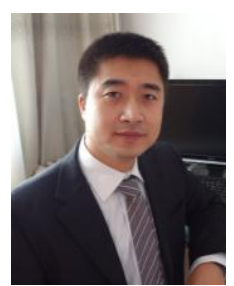

Yongfeng $\mathbf{L i}$ was employed as a professor in the School of Chemical Engineering in China University of Petroleum in Beijing after he was selected in the first "recruitment program of young global experts" in 2011. After his Ph.D in Dalian University of Technology, he had started to work in Japan since 2004. In the beginning, he worked as a postdoctoral 21 st COE research fellow in the department of electronic engineering of Tohoku University in Sendai/Japan from Nov. 2004. During Nov. 2006- Nov. 2008 he started to work as a JSPS (Japan Society for the Promotion of Science) research fellow. After that, he was employed as an assistant professor in this department and continued to work up to 2011. Now he starts to work as a full professor in China University of Petroleum in Beijing. He mainly performed the research of interdisciplinary area which consists of plasma science/engineering and nano-material science and engineering. He has published over 100 papers. 